\title{
FIRST PASSAGE TIME OF SKEW BROWNIAN MOTION
}

\author{
THILANKA APPUHAMILLAGE* AND \\ DANIEL SHELDON, ${ }^{* *}$ Oregon State University
}

\begin{abstract}
Nearly fifty years after the introduction of skew Brownian motion by Itô and McKean (1963), the first passage time distribution remains unknown. In this paper we first generalize results of Pitman and Yor (2011) and Csáki and Hu (2004) to derive formulae for the distribution of ranked excursion heights of skew Brownian motion, and then use these results to derive the first passage time distribution.
\end{abstract}

Keywords: Skew Brownian motion; ranked excursion height; first passage time

2010 Mathematics Subject Classification: Primary 60G99

Secondary $60 \mathrm{H} 99$

\section{Introduction}

In this paper we obtain formulae for the distributions of the first passage time and ranked excursion heights of skew Brownian motion. Since Itô and McKean (1963) first introduced skew Brownian motion, numerous papers in the mathematics literature have highlighted the special structure of the process. For example, Walsh (1978) showed that skew Brownian motion is a linear diffusion process with discontinuous scale and speed densities, and computed its transition probability density function. Harrison and Shepp (1981) showed that skew Brownian motion is the weak limit of a symmetric random walk on the integers with modified behavior at the origin, and also proved that it is the unique strong solution to the stochastic differential equation $\mathrm{d} X_{t}=\mathrm{d} B_{t}+(2 \alpha-1) \mathrm{d} L_{t}^{0}(X)$, where $B$ is Brownian motion and $L^{0}(X)$ is the symmetric local time of $X$ at 0 . Later LeGall (1984) and Ouknine (1990) extended the theory of strong solutions to the corresponding stochastic differential equation for a more general class of processes whose transition semigroups have functional-valued (generalized) infinitesimal generators. More recently, Appuhamillage et al. (2011) computed the trivariate density of the position, occupation, and local time of skew Brownian motion. Ramirez (2011) extended the notion of skew Brownian motion to multiskewed Brownian motion, a process whose local time is concentrated on a set of points.

Skew Brownian motion has also emerged in connection with diverse applications, including mathematical finance (see Decamps et al. (2006)), Monte Carlo simulation schemes (see Lejay and Martinez (2006)), and dispersion in heterogeneous media (see Ramirez et al. (2006), (2008)).

\footnotetext{
Received 8 October 2010; revision received 22 March 2012.

* Postal address: Department of Mathematics, Oregon State University, Corvallis, OR 97331, USA.

Email address: ireshara@math.oregonstate.edu

Research supported in part by the grant CMG EAR0724865 from the National Science Foundation.

** Postal address: School of Electrical Engineering and Computer Science, Oregon State University, Corvallis, OR 97331, USA. Email address: sheldon@eecs.oregonstate.edu

Research supported in part by the grant DBI-0905885 from the National Science Foundation.
} 
Skew Brownian motion is broadly applicable to diffusion problems in which the diffusion coefficient is discontinuous in space. For example, Appuhamillage et al. (2010) analyzed mathematical properties of skew Brownian motion as they relate to the convection-dispersion movement of solute through porous media in the presence of a sharp interface. Their work was motivated by experiments in hydrology demonstrating a fundamental asymmetry in the breakthrough curves of solute crossing a sharp interface in opposite directions (see Berkowitz et al. (2009)). In this context, the first passage time distribution of skew Brownian motion describes the breakthrough times of solute injected on one side of an interface and observed on the other side (see Appuhamillage et al. (2011)).

Similar situations arise in physical and natural sciences. For example, Brownian motion has been widely used (and also critiqued) as a model of animal movement (see Turchin (1996) and Blackwell (1997)). There is an emerging consensus among ecologists that landscape heterogeneity is a necessary ingredient of movement models (see Dalziel et al. (2008)). In particular, sharp interfaces delimiting two different movement regimes occur at the boundaries of habitat patches (see Schultz and Crone (2001), Turchin (1991), and Wiens and Milne (1989)), or at other environmental discontinuities such as those in ocean temperatures (see Pinaud et al. (2005)) or the level of surface chlorophyll in oceans (see Polovina et al. (2001)). Skew Brownian motion is an appropriate way to model this type of heterogeneity in animal movement models, and the first passage time is a quantity of biological interest: McKenzie et al. (2009) advocated the use of the first passage time to model the time required for an organism to first reach a specified location in the landscape (see also Fauchald and Tveraa (2003)), and gave as an example the foraging behavior of a predator searching for stationary prey items.

The main contribution of the present paper is to derive the first passage time distribution of skew Brownian motion. We achieve this by first characterizing the distribution of ranked excursion heights of skew Brownian motion. This result generalizes formulae of Pitman and Yor (2011) for the distribution of ranked excursion heights of the standard Brownian bridge, and analogous formulae presented in Csáki and $\mathrm{Hu}$ (2004) for Brownian motion. We then apply our formulae for the ranked excursion heights of skew Brownian motion to derive our main result on the first passage time.

The paper is organized as follows. In Section 2 we state the main results. In Section 3 we develop a coupled construction for two different skew Brownian motion processes with different skew parameters that leads to an important relationship between distributions of ranked excursion heights of the two processes, stated in Theorem 3.1. In Section 4 we prove the main results as corollaries to Theorem 3.1. In Section 5 we present several examples that demonstrate the calculations that are possible using the first passage time density, and also the asymmetry inherent in the first passage time of particles crossing an interface in opposite directions.

\section{Preliminaries and main results}

To set some notation and basic definitions, let $B=\left\{B_{t}: t \geq 0\right\}$ be the standard Brownian motion process on a probability space $(\Omega, \mathcal{F}, \mathrm{P})$ and let $J_{1}, J_{2}, \ldots$ denote the excursion intervals of the reflected process $\left\{\left|B_{t}\right|: t \geq 0\right\}$. For $\alpha \in(0,1)$, let $\left\{A_{m}^{(\alpha)}: m=0,1, \ldots\right\}$ be a sequence of independent and identically distributed (i.i.d.) \pm 1 Bernoulli random variables with $\mathrm{P}\left(A_{m}^{(\alpha)}=\right.$ $1)=\alpha$. Define the $\alpha$-skew Brownian motion process $B^{(\alpha)}$ started at 0 by

$$
B_{t}^{(\alpha)}=\sum_{m=1}^{\infty} \mathbf{1}_{J_{m}}(t) A_{m}^{(\alpha)}\left|B_{t}\right|,
$$

where $\mathbf{1}_{S}$ denotes the indicator function of the set $S$. 
Now let $M_{1}^{(\alpha)}(t) \geq M_{2}^{(\alpha)}(t) \geq \cdots \geq 0$ be the ranked decreasing sequence of excursion heights $\sup _{s \in J_{m} \cap[0, t]} B_{s}^{(\alpha)}$ ranging over all $m$ such that $J_{m} \cap[0, t]$ is nonempty. Note that a negative excursion has height 0 , and that the height of the final excursion is included in the ranked list even if that excursion is incomplete. Our first main result gives the distribution of ranked excursion heights.

Theorem 2.1. Fix $y \geq 0$ and $t>0$. Then, for each $j=1,2, \ldots$, the distribution of $M_{j}^{(\alpha)}(t)$ is given by the formula

$$
\mathrm{P}_{0}\left(M_{j}^{(\alpha)}(t)>y\right)=\sum_{h=1}^{\infty} 2\left(\begin{array}{c}
h-1 \\
j-1
\end{array}\right)(1-2 \alpha)^{h-j}(2 \alpha)^{j}\left(1-\Phi\left(\frac{(2 h-1) y}{\sqrt{t}}\right)\right),
$$

where $\Phi(\cdot)$ is the standard normal distribution function.

Now let $T_{y}^{(\alpha)}=\inf \left\{s \geq 0: B_{s}^{(\alpha)}=y\right\}$ denote the first time for $\alpha$-skew Brownian motion to reach $y$, and let $f^{(\alpha)}(x, y, t)$ denote the first passage time density to $y$ at time $t$ of $\alpha$-skew Brownian motion started at $x$. When $\alpha=\frac{1}{2}$, this is the well-known first passage time density $f(x, y, t)$ for Brownian motion (see, e.g. Bhattacharya and Waymire (2009, p. 30)):

$$
f^{(1 / 2)}(x, y, t) \equiv f(x, y, t)=\frac{|y-x|}{\sqrt{2 \pi} t^{3 / 2}} \exp \left\{-\frac{(y-x)^{2}}{2 t}\right\} .
$$

Note that $f^{(\alpha)}(x, y, t) \mathrm{d} t=\mathrm{P}_{x}\left(T_{y}^{(\alpha)} \in \mathrm{d} t\right)$. Our second main result gives formulae for the first passage time density. It is obviously true, by symmetry, that $f^{(\alpha)}(x, y, t)=$ $f^{(1-\alpha)}(-x,-y, t)$; thus, we state the formulae only for $y>0$.

Theorem 2.2. Fix $t>0$. Then

$$
f^{(\alpha)}(x, y, t)= \begin{cases}g_{x, y}^{(\alpha)}(t) & \text { for } x \leq 0<y, \\ g_{x, y}^{(\alpha)}(t)+h_{x, y}(t) & \\ \quad-\sum_{n=1}^{\infty} \frac{2}{\pi n} \sin \left\{\frac{\pi(y-x) n}{y}\right\} g_{0, y}^{(\alpha)} * \kappa_{n, y}(t) & \text { for } 0<x<y, \\ f(x, y, t) & \text { for } 0<y<x,\end{cases}
$$

where

$$
g_{x, y}^{(\alpha)}=2 \alpha \sum_{j=1}^{\infty}(1-2 \alpha)^{j-1} \frac{|x-(2 j-1) y|}{\sqrt{2 \pi} t^{3 / 2}} \exp \left\{-\frac{(x-(2 j-1) y)^{2}}{2 t}\right\} \text { for } x<y,
$$

the quantity $\kappa_{n, y}(t)$ is the density at time $t$ of the exponential distribution with parameter $\lambda(n, y)=\pi^{2} n^{2} / 2 y^{2}$, and

$$
h_{x, y}(t)=\frac{\pi}{y^{2}} \sum_{n=1}^{\infty} n \exp \left\{-\frac{\pi^{2} n^{2} t}{2 y^{2}}\right\} \sin \left\{\frac{\pi(y-x) n}{y}\right\}
$$

is the well-known formula (see, e.g. Feller (1968, p. 296)) for the probability that Brownian motion started at $x$ reaches 0 before reaching $y$, and that this event occurs in the time interval $\mathrm{d} t$.

Interpretation. The third case in the first passage time density, when $0<y<x$, is the simplest: it follows from the fact that all paths starting at $x$ reach $y$ without hitting 0 and, hence, they are all Brownian motion paths. 
In the first case, when $x \leq 0<y$, then all paths must cross 0 and densities are obtained as a straightforward corollary of Theorem 2.1. When $\alpha<\frac{1}{2}$, we can interpret $g_{x, y}^{(\alpha)}(t)$ probabilistically as the first passage time of standard Brownian motion started at $x$ to the level $(2 J-1) y$, where $J$ is a geometric random variable with parameter $2 \alpha$. Here is an informal argument for this fact. When $\alpha<\frac{1}{2}$, it is possible to construct $B^{(\alpha)}$ from the standard Brownian motion process $B$ by flipping each positive excursion of $B$ to be negative independently with probability $2 \alpha$, so the overall probability of an excursion remaining positive is $\frac{1}{2} \times 2 \alpha=\alpha$. Each excursion of $B$ that reaches $y$ is a candidate to contain the first visit to $y$ in the modified process $B^{(\alpha)}$. Define

$$
\tau_{0}=0, \quad \sigma_{j}=\inf \left\{t \geq \tau_{j-1}: B(t)=y\right\}, \quad \tau_{j}=\inf \left\{t \geq \sigma_{j}: B(t)=0\right\}, \quad j=1,2, \ldots
$$

The time $\sigma_{j}$ marks the first crossing of $y$ in the $j$ th excursion that reaches $y$, and $\tau_{j}$ marks the end of the same excursion. It is clear that the first of these excursions that is not flipped down will contain the first visit to $y$ in the skew Brownian motion process, that is, $T_{y}^{(\alpha)}=\sigma_{J}$, where $J$ is a geometric random variable with parameter $2 \alpha$. We can then show by repeated application of the reflection principle that $\sigma_{j}$ has the same distribution as $T_{(2 j-1) y}$ : for each $j=1,2, \ldots$, reflect the Brownian motion process about $(2 j-1) y$ during the time interval $\left[\sigma_{j}, \tau_{j}\right]$, which has the effect of displacing the process by $2 y$ (see Figure 1).

Note that this direct probabilistic interpretation does not apply when $\alpha>\frac{1}{2}$ : indeed, in this case the series $g_{x, y}^{(\alpha)}(t)$ alternates in sign. However, this informal argument contains key elements of our proof, which relates the excursion heights of $B^{(\alpha)}$ to those of $B$ through a coupled construction. Although we do not directly use the reflection principle in our proofs, it is used to derived the formulae for the distribution of excursion heights of Brownian motion, which we do invoke.

The interpretation of the second case of Theorem 2.2 is the most complex. In this case, when $x$ lies between 0 and $y$, we must consider two types of path from $x$ to $y$ : those that cross 0 , and

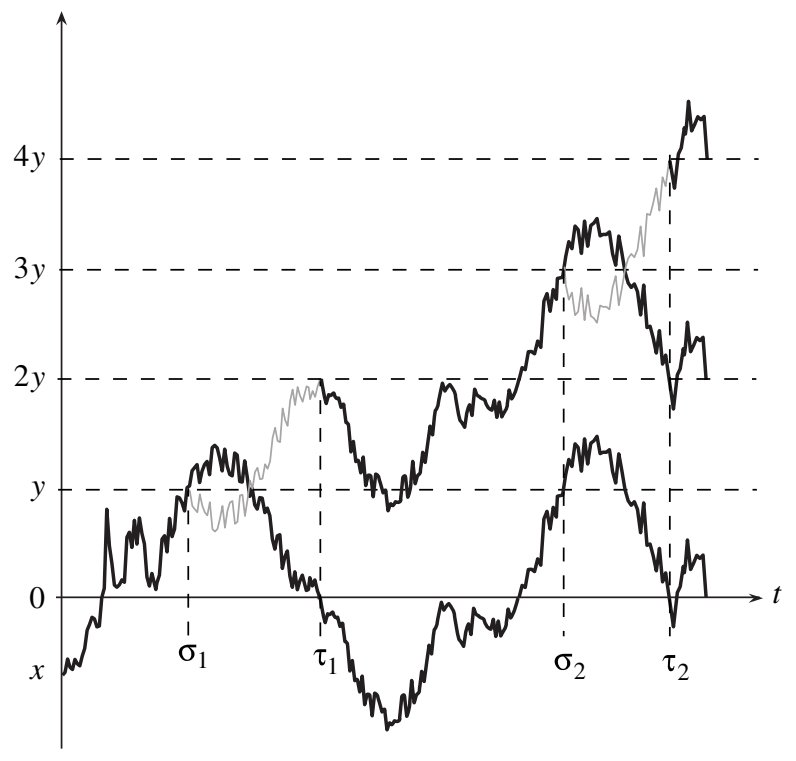

FIGURE 1: Probabilistic interpretation of Theorem 2.2. See text for explanation. 
those that reach $y$ before they reach 0 . We defer the details of the proof to Section 4 . After the initial preprint of this paper was published online (see Appuhamillage and Sheldon (2011)), Harada (2011) used different techniques to derive a formula for the second case of Theorem 2.2 that is slightly simpler than ours.

Note that, when $\alpha=\frac{1}{2}$, we recover existing results for standard Brownian motion. Namely, from Theorem 2.1 we recover the distribution of ranked excursion heights stated in Theorem 3.1 of Csáki and $\mathrm{Hu}$ (2004), and from Theorem 2.2 we recover the well-known first passage time distribution of standard Brownian motion (this fact is not immediately obvious, but nonetheless true, in the most complicated case when $0<x<y$ ).

\section{Relating excursion heights for $B^{(\alpha)}$ and $B^{(\beta)}$}

Let $0 \leq \alpha<\beta \leq 1$. Consider the following coupled construction of $\alpha$-skew and $\beta$-skew Brownian motions. Let $B$ be the standard Brownian motion process, and let $A^{(\beta)}=\left\{A_{m}^{(\beta)}: m=\right.$ $0,1, \ldots\}$ be independently chosen excursion signs so that (2.1) yields an instance of $\beta$-skew Brownian motion.

Next, let $\left\{A_{m}^{(\alpha / \beta)}: m=0,1, \ldots\right\}$ be a sequence of i.i.d. \pm 1 Bernoulli random variables independent of $A^{(\beta)}$ and $B$ with $\mathrm{P}\left(A_{m}^{(\alpha / \beta)}=1\right)=\alpha / \beta$. Define $A_{m}^{(\alpha)}$ as

$$
A_{m}^{(\alpha)}= \begin{cases}1, & A_{m}^{(\beta)}=1, A_{m}^{(\alpha / \beta)}=1, \\ -1, & \text { otherwise. }\end{cases}
$$

By construction, the sequence $\left\{A_{m}^{(\alpha)}: m=0,1, \ldots\right\}$ consists of i.i.d. \pm 1 Bernoulli random variables that are independent of $B$ with $\mathrm{P}\left(A_{m}^{(\alpha)}=1\right)=\alpha$. Hence, by using the variables $A_{m}^{(\alpha)}$ as the excursion signs in (2.1), we obtain an instance $B^{(\alpha)}$ of $\alpha$-skew Brownian motion.

We think of this as a two-step process: first, construct $B^{(\beta)}$ by independently setting each excursion of $|B|$ to be positive with probability $\beta$; then, for each positive excursion of $B^{(\beta)}$, independently decide whether to keep it positive (with probability $\alpha / \beta$ ), or flip it to be negative (with probability $1-\alpha / \beta$ ).

The following theorem is motivated by this coupled construction.

Theorem 3.1. Fix $y \geq 0, t>0$, and $\alpha, \beta \in(0,1)$. For each $j=1,2, \ldots$, the following relation between ranked excursion heights of $\alpha$ - and $\beta$-skew Brownian motions holds:

$$
\mathrm{P}_{0}\left(M_{j}^{(\alpha)}(t)>y\right)=\sum_{h=1}^{\infty}\left(\begin{array}{c}
h-1 \\
j-1
\end{array}\right)\left(1-\frac{\alpha}{\beta}\right)^{h-j}\left(\frac{\alpha}{\beta}\right)^{j} \mathrm{P}_{0}\left(M_{h}^{(\beta)}(t)>y\right) .
$$

Before proving Theorem 3.1, we state the following lemma from Pitman and Yor (2011), which we will use in the proof.

Lemma 3.1. (Pitman and Yor (2011, Lemma 9).) Let

$$
b_{k}=\sum_{m=0}^{\infty}\left(\begin{array}{l}
m \\
k
\end{array}\right) a_{m}, \quad k=0,1, \ldots,
$$

be the binomial moments of a nonnegative sequence $\left(a_{m}, m=0,1, \ldots\right)$. Let $B(\theta):=$ $\sum_{k=0}^{\infty} b_{k} \theta^{k}$, and suppose that $B\left(\theta_{1}\right)<\infty$ for some $\theta_{1}>1$. Then

$$
a_{m}=\sum_{k=0}^{\infty}(-1)^{k-m}\left(\begin{array}{l}
k \\
m
\end{array}\right) b_{k}, \quad m=0,1, \ldots,
$$

where the series is absolutely convergent. 
Proof of Theorem 3.1. For $\alpha<\beta$, we have, by the two-step construction of the excursion sign $A_{m}^{(\alpha)}, M_{j}^{(\alpha)}(t)=M_{H_{j}}^{(\beta)}(t)$, where $H_{j}$ has a negative binomial distribution:

$$
\mathrm{P}\left(H_{j}=h\right)=\left(\begin{array}{c}
h-1 \\
j-1
\end{array}\right)\left(1-\frac{\alpha}{\beta}\right)^{h-j}\left(\frac{\alpha}{\beta}\right)^{j}
$$

Hence,

$$
\mathrm{P}_{0}\left(M_{j}^{(\alpha)}(t)>y\right)=\sum_{h=1}^{\infty}\left(\begin{array}{c}
h-1 \\
j-1
\end{array}\right)\left(1-\frac{\alpha}{\beta}\right)^{h-j}\left(\frac{\alpha}{\beta}\right)^{j} \mathrm{P}_{0}\left(M_{h}^{(\beta)}(t)>y\right) .
$$

For $\beta<\alpha$, the relation can be inverted by an application of Lemma 3.1. Let $k:=j-1$ and $m:=h-1$. Then we can write (3.1) as

$$
\mathrm{P}_{0}\left(M_{k+1}^{(\alpha)}(t)>y\right)=\sum_{m=0}^{\infty}\left(\begin{array}{l}
m \\
k
\end{array}\right)\left(1-\frac{\alpha}{\beta}\right)^{m-k}\left(\frac{\alpha}{\beta}\right)^{k+1} \mathrm{P}_{0}\left(M_{m+1}^{(\beta)}(t)>y\right) .
$$

We then apply Lemma 3.1 to the sequences

$$
b_{k}:=\left(1-\frac{\alpha}{\beta}\right)^{k}\left(\frac{\alpha}{\beta}\right)^{-k-1} \mathrm{P}_{0}\left(M_{k+1}^{(\alpha)}(t)>y\right), \quad a_{m}:=\left(1-\frac{\alpha}{\beta}\right)^{m} \mathrm{P}_{0}\left(M_{m+1}^{(\beta)}(t)>y\right) .
$$

After simplifying, we obtain

$$
\mathrm{P}_{0}\left(M_{j}^{(\beta)}(t)>y\right)=\sum_{h=1}^{\infty}\left(\begin{array}{c}
h-1 \\
j-1
\end{array}\right)\left(1-\frac{\beta}{\alpha}\right)^{h-j}\left(\frac{\beta}{\alpha}\right)^{j} \mathrm{P}_{0}\left(M_{h}^{(\alpha)}(t)>y\right) .
$$

\section{Proofs of the main theorems}

We now observe that the main results announced in the introduction will follow as corollaries to Theorem 3.1. We first prove Theorem 2.1.

Proof of Theorem 2.1. By Theorem 3.1 of Csáki and Hu (2004),

$$
\mathrm{P}_{0}\left(M_{j}^{(1 / 2)}(t)>y\right)=2\left(1-\Phi\left((2 j-1) \frac{y}{\sqrt{t}}\right)\right) .
$$

The result is immediate from Theorem 3.1 by taking $\beta=\frac{1}{2}$ in (3.1).

We now use Theorem 2.1 and the following corollary to compute the distribution of the first passage time asserted in Theorem 2.2.

Corollary 4.1. Fix $t>0$. Then

$$
\begin{aligned}
& \mathrm{P}_{0}\left(T_{y}^{(\alpha)} \in \mathrm{d} t\right) \\
& \quad= \begin{cases}2 \alpha \sum_{h=1}^{\infty}(1-2 \alpha)^{h-1} \frac{(2 h-1) y}{\sqrt{2 \pi} t^{3 / 2}} \exp \left\{-\frac{((2 h-1) y)^{2}}{2 t}\right\} \mathrm{d} t & \text { for } y>0, \\
2(1-\alpha) \sum_{h=1}^{\infty}(2 \alpha-1)^{h-1} \frac{(2 h-1)(-y)}{\sqrt{2 \pi} t^{3 / 2}} \exp \left\{-\frac{((2 h-1) y)^{2}}{2 t}\right\} \mathrm{d} t & \text { for } y<0 .\end{cases}
\end{aligned}
$$


Proof. For $y>0$ and $t>0$, we have the following relation between the distributions of $T_{y}^{(\alpha)}$ and the highest excursion of skew Brownian motion started at 0 :

$$
\mathrm{P}_{0}\left(T_{y}^{(\alpha)}<t\right)=\mathrm{P}_{0}\left(M_{1}^{(\alpha)}(t)>y\right) .
$$

Thus, using Theorem 2.1, we have

$$
\begin{aligned}
\mathrm{P}_{0}\left(T_{y}^{(\alpha)}<t\right) & =\mathrm{P}_{0}\left(M_{1}^{(\alpha)}(t)>y\right) \\
& =4 \alpha \sum_{h=1}^{\infty}(1-2 \alpha)^{h-1} \int_{(2 h-1) y / \sqrt{t}}^{\infty} \frac{1}{\sqrt{2 \pi}} \exp \left\{-\frac{z^{2}}{2}\right\} \mathrm{d} z .
\end{aligned}
$$

The result is immediate after taking the derivative of the above expression with respect to $t$.

Proof of Theorem 2.2. Let $T_{y} \equiv T_{y}^{(1 / 2)}$ denote the first time standard Brownian motion reaches $y$. Recalling that $\mathrm{P}_{0}\left(T_{y} \in \mathrm{d} t\right)=\left(|y| / \sqrt{2 \pi} t^{3 / 2}\right) \exp \left\{-y^{2} / 2 t\right\} \mathrm{d} t$ (see, e.g. Bhattacharya and Waymire (2009, p. 30)), we can write (4.1) as

$$
\mathrm{P}_{0}\left(T_{y}^{(\alpha)} \in \mathrm{d} t\right)= \begin{cases}2 \alpha \sum_{h=1}^{\infty}(1-2 \alpha)^{h-1} \mathrm{P}_{0}\left(T_{(2 h-1) y} \in \mathrm{d} t\right) & \text { for } y>0, \\ 2(1-\alpha) \sum_{h=1}^{\infty}(2 \alpha-1)^{h-1} \mathrm{P}_{0}\left(T_{(2 h-1) y} \in \mathrm{d} t\right) & \text { for } y<0 .\end{cases}
$$

Now note that $T_{0}^{(\alpha)}$ is distributed as $T_{0}$ under $\mathrm{P}_{x}$ for $x \neq 0,0<\alpha<1$, and, thus, for $t>0$, we clearly have

$$
\mathrm{P}_{x}\left(T_{0}^{(\alpha)}>t\right)=\mathrm{P}_{x}\left(T_{0}>t\right) .
$$

Case 1: $x \leq 0<y$. Using the strong Markov property of skew Brownian motion,

$$
\mathrm{P}_{x}\left(T_{y}^{(\alpha)}>t\right)=\int_{0}^{t} \mathrm{P}_{x}\left(T_{0}>t-s\right) \mathrm{P}_{0}\left(T_{y}^{(\alpha)} \in \mathrm{d} s\right) .
$$

Then from the first case of (4.2) we have

$$
\begin{aligned}
\mathrm{P}_{x}\left(T_{y}^{(\alpha)}>t\right) & =2 \alpha \sum_{h=1}^{\infty}(1-2 \alpha)^{h-1} \int_{0}^{t} \mathrm{P}_{x}\left(T_{0}>t-s\right) \mathrm{P}_{0}\left(T_{(2 h-1) y} \in \mathrm{d} s\right) \\
& =2 \alpha \sum_{h=1}^{\infty}(1-2 \alpha)^{h-1} \mathrm{P}_{x}\left(T_{(2 h-1) y}>t\right) .
\end{aligned}
$$

By differentiating the above expression with respect to $t$ and recalling that $\mathrm{P}_{x}\left(T_{y} \in \mathrm{d} t\right)=$ $\left(|y-x| / \sqrt{2 \pi t^{3 / 2}}\right) \exp \left\{-(y-x)^{2} / 2 t\right\} \mathrm{d} t$, we have

$$
\mathrm{P}_{x}\left(T_{y}^{(\alpha)} \in \mathrm{d} t\right)=2 \alpha \sum_{h=1}^{\infty}(1-2 \alpha)^{h-1} \frac{|x-(2 h-1) y|}{\sqrt{2 \pi} t^{3 / 2}} \exp \left\{-\frac{(x-(2 h-1) y)^{2}}{2 t}\right\} \mathrm{d} t .
$$

Case 2: $0<x<y$. Observe that

$$
\mathrm{P}_{x}\left(T_{y}^{(\alpha)} \in \mathrm{d} t\right)=\mathrm{P}_{x}\left(T_{y}^{(\alpha)} \in \mathrm{d} t,\left(T_{0}^{(\alpha)} \leq t\right)\right)+\mathrm{P}_{x}\left(T_{y}^{(\alpha)} \in \mathrm{d} t,\left(T_{0}^{(\alpha)}>t\right)\right) .
$$

We recall the following formula (see, e.g. Feller (1968, p. 296)) that we will use to compute (4.3):

$$
\mathrm{P}_{x}\left(T_{0} \in \mathrm{d} t,\left(T_{y}>t\right)\right)=\frac{\pi}{y^{2}} \sum_{n=1}^{\infty} n \exp \left\{-\frac{\pi^{2} n^{2} t}{2 y^{2}}\right\} \sin \left\{\frac{\pi x n}{y}\right\} \mathrm{d} t .
$$


Since the skew Brownian motion is Brownian motion until it reaches 0 for the first time and from the reflection principle of Brownian motion, we can write the second term on the right-hand side of (4.3) using (4.4) as

$$
\begin{aligned}
\mathrm{P}_{x}\left(T_{y}^{(\alpha)} \in \mathrm{d} t,\left(T_{0}^{(\alpha)}>t\right)\right) & =\mathrm{P}_{x}\left(T_{y} \in \mathrm{d} t,\left(T_{0}>t\right)\right) \\
& =\mathrm{P}_{y-x}\left(T_{0} \in \mathrm{d} t,\left(T_{y}>t\right)\right) \\
& =\frac{\pi}{y^{2}} \sum_{n=1}^{\infty} n \exp \left\{-\frac{\pi^{2} n^{2} t}{2 y^{2}}\right\} \sin \left\{\frac{\pi(y-x) n}{y}\right\} \mathrm{d} t .
\end{aligned}
$$

For the first term on the right-hand side of (4.3), note that

$$
\begin{aligned}
\mathrm{P}_{x}\left(T_{0}^{(\alpha)}<T_{y}^{(\alpha)}<t\right) \\
=\mathrm{E}_{x}\left[\mathbf{1}_{\left\{T_{0}^{(\alpha)}<T_{y}^{(\alpha)}<t\right\}}\right] \\
=\mathrm{E}_{x}\left[\mathrm{E}_{x}\left[\mathbf{1}_{\left\{T_{0}^{(\alpha)}<T_{y}^{(\alpha)}<t\right\}} \mid T_{0}^{(\alpha)}, \mathbf{1}_{\left\{T_{0}^{(\alpha)}<T_{y}^{(\alpha)}\right\}}\right]\right] \\
=\int_{0}^{t} \mathrm{E}_{x}\left[\mathbf{1}_{\left\{T_{0}^{(\alpha)}<T_{y}^{(\alpha)}<t\right\}} \mid T_{0}^{(\alpha)}=s, \mathbf{1}_{\left\{T_{0}^{(\alpha)}<T_{y}^{(\alpha)}\right\}}\right] \mathrm{P}_{x}\left(T_{0}^{(\alpha)} \in \mathrm{d} s,\left(T_{0}^{(\alpha)}<T_{y}^{(\alpha)}\right)\right) \\
\quad+\int_{0}^{t} \mathrm{E}_{x}\left[\mathbf{1}_{\left\{T_{0}^{(\alpha)}<T_{y}^{(\alpha)}<t\right\}} \mid T_{0}^{(\alpha)}=s, \mathbf{1}_{\left\{T_{0}^{(\alpha)} \geq T_{y}^{(\alpha)}\right\}}\right] \mathrm{P}_{x}\left(T_{0}^{(\alpha)} \in \mathrm{d} s,\left(T_{0}^{(\alpha)} \geq T_{y}^{(\alpha)}\right)\right) .
\end{aligned}
$$

Using the strong Markov property of skew Brownian motion and the fact that

$$
\mathrm{E}_{x}\left[\mathbf{1}_{\left\{T_{0}^{(\alpha)}<T_{y}^{(\alpha)}<t\right\}} \mid T_{0}^{(\alpha)}=s, \mathbf{1}_{\left\{T_{0}^{(\alpha)} \geq T_{y}^{(\alpha)}\right\}}\right]=0,
$$

we have

$$
\begin{aligned}
\mathrm{P}_{x}\left(T_{0}^{(\alpha)}<T_{y}^{(\alpha)}<t\right) & =\int_{0}^{t} \mathrm{E}_{0}\left[\mathbf{1}_{\left\{T_{y}^{(\alpha)}<t-s\right\}}\right] \mathrm{P}_{x}\left(T_{0}^{(\alpha)} \in \mathrm{d} s,\left(T_{0}^{(\alpha)}<T_{y}^{(\alpha)}\right)\right) \\
& =\int_{0}^{t} \mathrm{P}_{0}\left(T_{y}^{(\alpha)}<t-s\right) \mathrm{P}_{x}\left(T_{0}^{(\alpha)} \in \mathrm{d} s,\left(T_{0}^{(\alpha)}<T_{y}^{(\alpha)}\right)\right) .
\end{aligned}
$$

Using (4.2) and again from the fact that the skew Brownian motion is Brownian motion until it reaches 0 for the first time, and $\mathrm{P}_{x}\left(T_{0}^{(\alpha)}<T_{y}^{(\alpha)}\right)=\mathrm{P}_{x}\left(T_{0}<T_{y}\right.$ ) (note that here $0<x<y$ ), we have

$$
\begin{aligned}
\mathrm{P}_{x}\left(T_{0}^{(\alpha)}<T_{y}^{(\alpha)}<t\right) \\
=\int_{0}^{t} 2 \alpha \sum_{h=1}^{\infty}(1-2 \alpha)^{h-1} \mathrm{P}_{0}\left(T_{(2 h-1) y}<t-s\right) \mathrm{P}_{x}\left(T_{0} \in \mathrm{d} s,\left(T_{0}<T_{y}\right)\right) \\
=2 \alpha \sum_{h=1}^{\infty}(1-2 \alpha)^{h-1} \int_{0}^{t} \mathrm{P}_{0}\left(T_{(2 h-1) y}<t-s\right) \mathrm{P}_{x}\left(T_{0} \in \mathrm{d} s,\left(T_{0}<T_{y}\right)\right) \\
=2 \alpha \sum_{h=1}^{\infty}(1-2 \alpha)^{h-1} \int_{0}^{t} \mathrm{P}_{0}\left(T_{(2 h-1) y}<t-s\right) \mathrm{P}_{x}\left(T_{0} \in \mathrm{d} s\right) \\
\quad-2 \alpha \sum_{h=1}^{\infty}(1-2 \alpha)^{h-1} \int_{0}^{t} \mathrm{P}_{0}\left(T_{(2 h-1) y}<t-s\right) \mathrm{P}_{x}\left(T_{0} \in \mathrm{d} s,\left(T_{0}>T_{y}\right)\right)
\end{aligned}
$$




$$
\begin{aligned}
= & 2 \alpha \sum_{h=1}^{\infty}(1-2 \alpha)^{h-1} \mathrm{P}_{x}\left(T_{(2 h-1) y}<t\right) \\
& -2 \alpha \sum_{h=1}^{\infty}(1-2 \alpha)^{h-1} \int_{0}^{t} \mathrm{P}_{0}\left(T_{(2 h-1) y}<t-s\right) \mathrm{P}_{x}\left(T_{0} \in \mathrm{d} s,\left(T_{0}>T_{y}\right)\right) .
\end{aligned}
$$

For $h \geq 1$, the convolution integral in the sum of the second term in the above equation can be written using (4.5) as

$$
\begin{aligned}
& \int_{0}^{t} \mathrm{P}_{0}\left(T_{(2 h-1) y}<t-s\right) \mathrm{P}_{x}\left(T_{0} \in \mathrm{d} s,\left(T_{0}>T_{y}\right)\right) \\
&=\int_{0}^{t} \mathrm{P}_{x}\left(T_{0}<t-s,\left(T_{0}>T_{y}\right)\right) \mathrm{P}_{0}\left(T_{(2 h-1) y} \in \mathrm{d} s\right) \\
&=\int_{0}^{t} \sum_{n=1}^{\infty} \frac{2}{\pi n} \sin \left\{\frac{\pi(y-x) n}{y}\right\} \mathrm{P}_{0}\left(T_{(2 h-1) y} \in \mathrm{d} s\right) \\
&-\int_{0}^{t} \sum_{n=1}^{\infty} \frac{2}{\pi n} \exp \left\{-\frac{\pi^{2} n^{2}(t-s)}{2 y^{2}}\right\} \sin \left\{\frac{\pi(y-x) n}{y}\right\} \mathrm{P}_{0}\left(T_{(2 h-1) y} \in \mathrm{d} s\right) \\
&= \sum_{n=1}^{\infty} \frac{2}{\pi n} \sin \left\{\frac{\pi(y-x) n}{y}\right\} \mathrm{P}_{0}\left(T_{(2 h-1) y}<t\right) \\
&-\sum_{n=1}^{\infty} \frac{2}{\pi n} \exp \left\{-\frac{\pi^{2} n^{2} t}{2 y^{2}}\right\} \sin \left\{\frac{\pi(y-x) n}{y}\right\} \int_{0}^{t} \exp \left\{\frac{\pi^{2} n^{2} s}{2 y^{2}}\right\} \mathrm{P}_{0}\left(T_{(2 h-1) y} \in \mathrm{d} s\right) .
\end{aligned}
$$

Then we have

$$
\begin{aligned}
\mathrm{P}_{x}\left(T_{0}^{(\alpha)}<T_{y}^{(\alpha)}<t\right)= & 2 \alpha \sum_{h=1}^{\infty}(1-2 \alpha)^{h-1} \mathrm{P}_{x}\left(T_{(2 h-1) y}<t\right) \\
& -2 \alpha \sum_{h=1}^{\infty} \sum_{n=1}^{\infty}(1-2 \alpha)^{h-1} \frac{2}{\pi n} \sin \left\{\frac{\pi(y-x) n}{y}\right\} \mathrm{P}_{0}\left(T_{(2 h-1) y}<t\right) \\
& +2 \alpha \sum_{h=1}^{\infty} \sum_{n=1}^{\infty}(1-2 \alpha)^{h-1} \frac{2}{\pi n} \exp \left\{-\frac{\pi^{2} n^{2} t}{2 y^{2}}\right\} \sin \left\{\frac{\pi(y-x) n}{y}\right\} \\
& \times \int_{0}^{t} \exp \left\{\frac{\pi^{2} n^{2} s}{2 y^{2}}\right\} \mathrm{P}_{0}\left(T_{(2 h-1) y} \in \mathrm{d} s\right) .
\end{aligned}
$$

By differentiating the above equation with respect to $t$ we obtain

$$
\begin{aligned}
\mathrm{P}_{x}\left(T_{y}^{(\alpha)} \in \mathrm{d} t,\left(T_{0}^{(\alpha)} \leq T_{y}^{(\alpha)}\right)\right) \\
=2 \alpha \sum_{h=1}^{\infty}(1-2 \alpha)^{h-1} \mathrm{P}_{x}\left(T_{(2 h-1) y} \in \mathrm{d} t\right) \\
\quad-2 \alpha \sum_{h=1}^{\infty} \sum_{n=1}^{\infty}(1-2 \alpha)^{h-1} \frac{2}{\pi n} \sin \left\{\frac{\pi(y-x) n}{y}\right\} \mathrm{P}_{0}\left(T_{(2 h-1) y} \in \mathrm{d} t\right)
\end{aligned}
$$




$$
\begin{aligned}
+2 \alpha \sum_{h=1}^{\infty} \sum_{n=1}^{\infty}(1-2 \alpha)^{h-1} \frac{2}{\pi n} \exp \left\{-\frac{\pi^{2} n^{2} t}{2 y^{2}}\right\} \sin \left\{\frac{\pi(y-x) n}{y}\right\} \\
\quad \times \exp \left\{\frac{\pi^{2} n^{2} t}{2 y^{2}}\right\} \mathrm{P}_{0}\left(T_{(2 h-1) y} \in \mathrm{d} t\right) \\
-2 \alpha \sum_{h=1}^{\infty} \sum_{n=1}^{\infty}(1-2 \alpha)^{h-1} \frac{\pi n}{y^{2}} \exp \left\{-\frac{\pi^{2} n^{2} t}{2 y^{2}}\right\} \sin \left\{\frac{\pi(y-x) n}{y}\right\} \\
\times \int_{0}^{t} \exp \left\{\frac{\pi^{2} n^{2} s}{2 y^{2}}\right\} \mathrm{P}_{0}\left(T_{(2 h-1) y} \in \mathrm{d} s\right) \mathrm{d} t .
\end{aligned}
$$

Recalling that $\mathrm{P}_{x}\left(T_{y} \in \mathrm{d} t\right)=\left(|y-x| / \sqrt{2 \pi} t^{3 / 2}\right) \exp \left\{-(y-x)^{2} / 2 t\right\} \mathrm{d} t$, and by (4.3), (4.5), and (4.6), we have

$$
\begin{aligned}
& \mathrm{P}_{x}\left(T_{y}^{(\alpha)} \in \mathrm{d} t\right)=2 \alpha \sum_{h=1}^{\infty}(1-2 \alpha)^{h-1} \frac{|x-(2 h-1) y|}{\sqrt{2 \pi} t^{3 / 2}} \exp \left\{-\frac{(x-(2 h-1) y)^{2}}{2 t}\right\} \mathrm{d} t \\
& -2 \alpha \sum_{h=1}^{\infty} \sum_{n=1}^{\infty}(1-2 \alpha)^{h-1} \frac{\pi n}{y^{2}} \exp \left\{-\frac{\pi^{2} n^{2} t}{2 y^{2}}\right\} \sin \left\{\frac{\pi(y-x) n}{y}\right\} \\
& \times \int_{0}^{t} \exp \left\{\frac{\pi^{2} n^{2} s}{2 y^{2}}\right\} \frac{(2 h-1) y}{\sqrt{2 \pi} s^{3 / 2}} \exp \left\{-\frac{((2 h-1) y)^{2}}{2 s}\right\} \mathrm{d} s \mathrm{~d} t \\
& +\frac{\pi}{y^{2}} \sum_{n=1}^{\infty} n \exp \left\{-\frac{\pi^{2} n^{2} t}{2 y^{2}}\right\} \sin \left\{\frac{\pi(y-x) n}{y}\right\} \mathrm{d} t \\
& =2 \alpha \sum_{h=1}^{\infty}(1-2 \alpha)^{h-1} \frac{|x-(2 h-1) y|}{\sqrt{2 \pi} t^{3 / 2}} \exp \left\{-\frac{(x-(2 h-1) y)^{2}}{2 t}\right\} \mathrm{d} t \\
& -\sum_{n=1}^{\infty} \frac{2}{\pi n} \sin \left\{\frac{\pi(y-x) n}{y}\right\} \\
& \times \int_{0}^{t} \frac{\pi^{2} n^{2}}{2 y^{2}} \exp \left\{-\frac{\pi^{2} n^{2}(t-s)}{2 y^{2}}\right\} 2 \alpha \\
& \times \sum_{h=1}^{\infty}(1-2 \alpha)^{h-1} \frac{(2 h-1) y}{\sqrt{2 \pi} s^{3 / 2}} \exp \left\{-\frac{((2 h-1) y)^{2}}{2 t}\right\} \mathrm{d} s \mathrm{~d} t \\
& +\frac{\pi}{y^{2}} \sum_{n=1}^{\infty} n \exp \left\{-\frac{\pi^{2} n^{2} t}{2 y^{2}}\right\} \sin \left\{\frac{\pi(y-x) n}{y}\right\} \mathrm{d} t .
\end{aligned}
$$

Case 3: $0<y<x$. Note that, in this case all skew Brownian motion paths till the first passage time to $y$ are away from 0 . Thus, we have

$$
\mathrm{P}_{x}\left(T_{y}^{(\alpha)} \in \mathrm{d} t\right)=\frac{|y-x|}{\sqrt{2 \pi} t^{3 / 2}} \exp \left\{-\frac{(y-x)^{2}}{2 t}\right\} \mathrm{d} t .
$$

\section{Asymmetries in the first passage time density}

A basic property of the first passage time density of regular Brownian motion is that it is symmetric in $x$ and $y$, i.e. that $\mathrm{P}_{x}\left(T_{y} \in \mathrm{d} t\right)=\mathrm{P}_{y}\left(T_{x} \in \mathrm{d} t\right)$ for any $x, y \in \mathbb{R}$ and $t \geq 0$. A practically important feature of skew Brownian motion is that it introduces an asymmetry in 

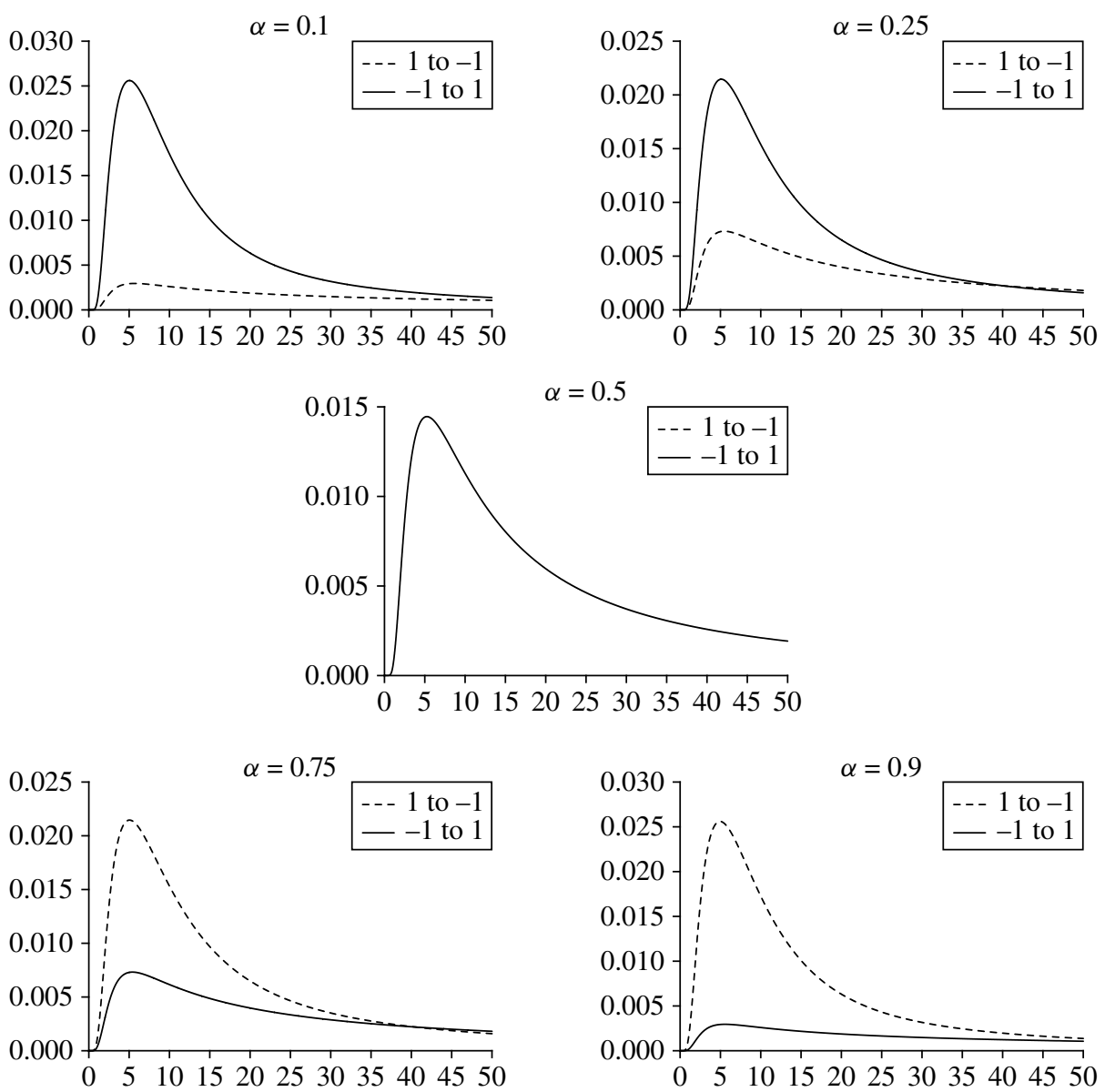

Figure 2: The densities $\mathrm{P}_{-1}\left(T_{1}^{(\alpha)} \in \mathrm{d} t\right)$ (solid lines) and $\mathrm{P}_{1}\left(T_{-1}^{(\alpha)} \in \mathrm{d} t\right.$ ) (dashed lines) for $\alpha<\frac{1}{2}$ (top row), $\alpha=\frac{1}{2}$ (middle row), and $\alpha>\frac{1}{2}$ (bottom row).

the first passage time density for $x$ and $y$ on opposite sides of the origin. Appuhamillage et al. (2011) first demonstrated this by proving that there is a stochastic ordering between the random variables with densities $\mathrm{P}_{-y}\left(T_{y}^{(\alpha)} \in \mathrm{d} t\right)$ and $\mathrm{P}_{y}\left(T_{-y}^{(\alpha)} \in \mathrm{d} t\right)$ when $\alpha \neq \frac{1}{2}$. In simple terms, it takes longer to cross from negative to positive than to cross from positive to negative when $\alpha<\frac{1}{2}$, and the opposite is true when $\alpha>\frac{1}{2}$.

Our results quantify these relationships further by explicitly giving the densities in each case. We conclude by illustrating the numerically computed densities $\mathrm{P}_{-1}\left(T_{1}^{(\alpha)} \in \mathrm{d} t\right)$ and $\mathrm{P}_{1}\left(T_{-1}^{(\alpha)} \in \mathrm{d} t\right)$ for different values of $\alpha$. In Figure $2\left(\right.$ a) we see that $\mathrm{P}_{-1}\left(T_{1}^{(\alpha)} \in \mathrm{d} t\right)<\mathrm{P}_{1}\left(T_{-1}^{(\alpha)} \in\right.$ $\mathrm{d} t$ ) for $\alpha<\frac{1}{2}$. In Figure 2(b), we recover the symmetry of Brownian motion when $\alpha=\frac{1}{2}$. In Figure 2(c) we see that $\mathrm{P}_{-1}\left(T_{1}^{(\alpha)} \in \mathrm{d} t\right)>\mathrm{P}_{1}\left(T_{-1}^{(\alpha)} \in \mathrm{d} t\right)$ for $\alpha>\frac{1}{2}$.

\section{Acknowledgements}

The authors wish to express their appreciation to Professor Enrique Thomann, Professor Edward Waymire, and Professor Julia Jones for the great interest they showed in this work, and for their fruitful comments and useful advice. 


\section{References}

Appuhamillage, T. and Sheldon, D. (2011). First passage time of skew Brownian motion. Preprint. Available at http://arxiv.org/abs/1008.2989v2.

Appuhamillage, T. A. et al. (2010). Solute transport across an interface: a Fickian theory for skewness in breakthrough curves. Water Resources Res. 46, 13 pp.

Appuhamillage, T. et al. (2011). Occupation and local times for skew Brownian motion with applications to dispersion across an interface. Ann. Appl. Prob. 21, 183-214.

Berkowitz, B., Cortis, A., Dror, I. AND Scher, H. (2009). Laboratory experiments on dispersive transport across interfaces: the role of flow direction. Water Resources Res. 45, 6 pp.

Bhattacharya, R. N. and Waymire, E. C. (2009). Stochastic Processes with Applications (SIAM Classics Appl. Math. 61). Society for Industrial and Applied Mathematics, Philadelphia, PA.

Blackwell, P. G. (1997). Random diffusion models for animal movement. Ecological Modelling 100, 87-102.

CsáKI, E. AND Hu, Y. (2004). Invariance principles for ranked excursion lengths and heights. Electron. Commun. Prob. 9, 14-21.

Dalziel, B. D., Morales, J. M. and Fryxell, J. M. (2008). Fitting probability distributions to animal movement trajectories: using artificial neural networks to link distance, resources, and memory. Amer. Naturalist 172, 248-258.

Decamps, M., Goovaerts, M. And Schoutens, W. (2006). Asymmetric skew Bessel processes and their applications to finance. J. Comput. Appl. Math. 186, 130-147.

FAUChald, P. AND TVERAA, T. (2003). Using first-passage time in the analysis of area-restricted search and habitat selection. Ecology 84, 282-288.

Feller, W. (1968). An Introduction to Probability Theory and Its Applications, Vol. I, 3rd edn. John Wiley, New York.

Harada, S. J. (2011). Fundamental solution to the heat equation with a discontinuous diffusion coefficient and applications to skew Brownian motion and oceanography. Masters Thesis, Oregon State University.

Harrison, J. M. ANd Shepp, L. A. (1981). On skew Brownian motion. Ann. Prob. 9, 309-313.

Itô, K. AND McKean, H. P., JR. (1963). Brownian motions on a half line. Illinois J. Math. 7, 181-231.

LE GALL, J.-F. (1984). One-dimensional stochastic differential equations involving the local times of the unknown process. In Stochastic Analysis and Applications (Swansea, 1983; Lecture Notes Math. 1095), Springer, Berlin, pp. $51-82$.

Lejay, A. And Martinez, M. (2006). A scheme for simulating one-dimensional diffusion processes with discontinuous coefficients. Ann. Appl. Prob. 16, 107-139.

McKenzie, H. W., Lewis, M. A. And Merrill, E. H. (2009). First passage time analysis of animal movement and insights into the functional response. Bull. Math. Biol. 71, 107-129.

Ouknine, Y. (1990). Le "Skew-Brownian motion" et les processus qui en dérivent. Teor. Veroyat. Primen. 35, 173-179 (in French). English translation: Theory Prob. Appl. 35, 163-169.

Pinaud, D., Cherel, Y. AND Weimerskirch, H. (2005). Effect of environmental variability on habitat selection, diet, provisioning behaviour and chick growth in yellow-nosed albatrosses. Marine Ecology Progr. Ser. 298, $295-304$.

Pitman, J. And Yor, M. (2001). On the distribution of ranked heights of excursions of a Brownian bridge. Ann. Prob. 29, 361-384.

Polovina, J. J., Howell, E., Kobayashi, D. R. And SeKi, M. P. (2001). The transition zone chlorophyll front, a dynamic global feature defining migration and forage habitat for marine resources. Progr. Oceanography 49, 469-483.

Ramirez, J. M. (2011). Multi-skewed Brownian motion and diffusion in layered media. Proc. Amer. Math. Soc. 139, 3739-3752.

Ramirez, J. M. et al. (2006). A generalized Taylor-Aris formula and skew diffusion. Multiscale Model. Simul. 5, 786-801.

RAmirez, J. M. et al. (2008). A note on the theoretical foundations of particle tracking methods in heterogeneous porous media. Water Resources Res. 44, 5 pp.

Schultz, C. B. ANd Crone, E. E. (2001). Edge-mediated dispersal behavior in a prairie butterfly. Ecology 82, 18791892.

TurCHIN, P. (1991). Translating foraging movements in heterogeneous environments into the spatial distribution of foragers. Ecology 72, 1253-1266.

Turchin, P. (1996). Fractal analyses of animal movement: a critique. Ecology 77, 2086-2090.

Walsh, J. B. (1978). A diffusion with a discontinuous local time. Asterisque 52-53, 37-45.

Wiens, J. A. AND Milne, B. T. (1989). Scaling of 'landscapes' in landscape ecology, or, landscape ecology from a beetle's perspective. Landscape Ecology 3, 87-96. 